\title{
MODIFIED LENGTH SPECTRUM METRIC ON THE TEICHMÜLLER SPACE OF A RIEMANN SURFACE WITH BOUNDARY
}

\author{
Jun $\mathrm{Hu}$ and Francisco G. Jimenez-Lopez \\ Brooklyn College of CUNY, Department of Mathematics \\ Brooklyn, NY 11210, U.S.A.; junhu@brooklyn.cuny.edu \\ and Graduate Center of CUNY, Ph.D. Program in Mathematics \\ 365 Fifth Avenue, New York, NY 10016, U.S.A.; JHu1@gc.cuny.edu \\ CUNY Graduate Center, Department of Mathematics \\ 365 Fifth Avenue, New York, NY 10016, U.S.A.; fjimenez_lopez@gc.cuny.edu

\begin{abstract}
Let $S_{0}$ be a bordered Riemann surface of finite type, and let $T\left(S_{0}\right)$ (resp. $T^{R}\left(S_{0}\right)$ ) be the Teichmüller space (resp. reduced Teichmüller space) of $S_{0}$. The length spectrum function defines a metric on $T^{R}\left(S_{0}\right)$ but not on $T\left(S_{0}\right)$. In this paper, we introduce a modified length spectrum function that does define a metric on $T\left(S_{0}\right)$. Then we show that if two points of $T\left(S_{0}\right)$ are close in the Teichmüller metric then they are close in the modified length spectrum metric, but the converse is not true. We also prove that $T\left(S_{0}\right)$ is not complete under this modified length spectrum metric.
\end{abstract}

\section{Introduction}

Let $S_{0}$ be a Riemann surface. A marked Riemann surface is a pair $(S, f)$, where $f: S_{0} \rightarrow S$ is a quasiconformal mapping. Two pairs $\left(S_{1}, f_{1}\right)$ and $\left(S_{2}, f_{2}\right)$ are equivalent if there exists a conformal mapping $c: S_{1} \rightarrow S_{2}$ such that $c \circ f_{1}$ is homotopic to $f_{2}$. The reduced Teichmüller space $T^{R}\left(S_{0}\right)$ is the set of the equivalence classes $[S, f]$. Furthermore, $c \circ f_{1}$ is homotopic to $f_{2}$ relative to boundary if $c \circ f_{1}$ agrees with $f_{2}$ on the boundary and there is a homotopy between them such that it takes the same image at every point on the boundary when the other variable of the homotopy changes. The set of the equivalence classes $[S, f]$ under such a homotopy is called the Teichmüller space $T\left(S_{0}\right)$. Clearly, if $S_{0}$ has no boundary, then $T^{R}\left(S_{0}\right)=T\left(S_{0}\right)$.

The Teichmüller metric on $T^{R}\left(S_{0}\right)$ (resp. $T\left(S_{0}\right)$ ) is defined by

$$
d_{T}\left(\left[S_{1}, f_{1}\right],\left[S_{2}, f_{2}\right]\right)=\log K(f),
$$

where $f$ is an extremal quasiconformal mapping in the homotopy class (resp. the homotopy class relative to boundary) of $f_{2} \circ f_{1}^{-1}$ and $K(f)$ represents the maximal dilation of $f$.

By comparing hyperbolic lengths of closed curves and their images, another metric, called the length spectrum metric, is defined on reduced Teichmüller spaces. Let $S$ be a Riemann surface and $\Sigma_{S}^{\prime}$ a collection of nontrivial closed curves on $S$ such that none of them is homotopic to a puncture and no two of them are homotopic to each other. We assume that $\Sigma_{S}^{\prime}$ is maximal in the sense that every nontrivial closed curve

doi:10.5186/aasfm.2014.3945

2010 Mathematics Subject Classification: Primary 30F60.

Key words: Teichmüller space, Length Spectrum, Teichmüller metric. 
on $S$ that is not homotopic to a puncture is homotopic to an element of $\Sigma_{S}^{\prime}$. For any closed curve $\gamma$ on $S$, let $l_{S}(\gamma)$ denote the length of the geodesic in the homotopy class of $\gamma$ with respect to the hyperbolic metric. The length spectrum metric on $T^{R}\left(S_{0}\right)$ is defined by

$$
d_{L}\left(\left[S_{1}, f_{1}\right],\left[S_{2}, f_{2}\right]\right)=\log \sup _{\gamma \in \Sigma_{S_{1}}^{\prime}}\left\{\frac{l_{S_{2}}\left(f_{2} \circ f_{1}^{-1}(\gamma)\right)}{l_{S_{1}}(\gamma)}, \frac{l_{S_{1}}(\gamma)}{l_{S_{2}}\left(f_{2} \circ f_{1}^{-1}(\gamma)\right)}\right\} .
$$

This metric was introduced and studied by Sorvali [9] in 1972. In 1975, Sorvali [10] proved that the two metrics $d_{T}$ and $d_{L}$ are metrically equivalent on the Teichmüller space of a torus and posed a question as to whether or not this is true on the Teichmüller space of an arbitrary Riemann surface. In 1986, Li [5] showed that the two metrics induce the same topology on the Teichmüller spaces of compact Riemann surfaces. In 1999, Liu [7] generalized Li's result to the Teichmüller spaces of Riemann surfaces of finite topological type. Then in 2003, Li [6] gave a negative answer to Sorvali's question. In the same year, Shiga [8] proved that if $S_{0}$ is a Riemann surface of infinite topological type admitting a pants decomposition that is both upper-bounded and lower-bounded, then these two metrics define the same topology on $T^{R}\left(S_{0}\right)$. In the same paper, he also provided an example of a surface $S_{0}$ of infinite topological type such that on $T^{R}\left(S_{0}\right), d_{T}$ and $d_{L}$ are not topologically equivalent.

When a Riemann surface $S_{0}$ has boundary, $T^{R}\left(S_{0}\right) \neq T\left(S_{0}\right)$. In this case, $d_{L}$ does not define a metric on $T\left(S_{0}\right)$ since it does not separate points. For if $f: S_{0} \rightarrow S_{0}$ is given by a Dehn twist along a boundary geodesic $\beta$, i.e., $\beta$ is a geodesic homotopic to some boundary component of $S_{0}$, then $d_{T}\left(\left[S_{0}, f\right],\left[S_{0}, i d\right]\right)>0$ in $T\left(S_{0}\right)$. Thus $\left[S_{0}, f\right] \neq\left[S_{0}, i d\right]$ in $T\left(S_{0}\right)$. However, since there is no closed geodesic crossing $\beta$, the length of every closed geodesic $\gamma$ is not changed under the Dehn twist and then $d_{L}\left(\left[S_{0}, f\right],\left[S_{0}, i d\right]\right)=0$.

Assume that $S_{0}$ has a boundary. In this paper, we first introduce a modified length spectrum that does define a metric on $T\left(S_{0}\right)$. Then we study properties of this new metric and its relationship with the Teichmüller metric on $T\left(S_{0}\right)$ when $S_{0}$ is of finite topological type.

Let $S$ be a Riemann surface with boundary and $\Sigma_{S}^{\prime \prime}$ a collection of arcs connecting boundary components of $S$ such that none of them is homotopic to a boundary segment relative to endpoints and no two of them are homotopic to each other relative to endpoints. We assume that $\Sigma_{S}^{\prime \prime}$ is maximal in the sense that every arc connecting boundary components of $S$ that is not homotopic to a boundary segment relative to endpoints is homotopic to an element of $\Sigma_{S}^{\prime \prime}$ relative to endpoints. For any arc $\gamma$ joining two boundary components, there exists a unique geodesic arc $\alpha$ homotopic to $\gamma$ relative to endpoints. Let $\beta_{1}$ and $\beta_{2}$ be the two closed geodesics homotopic to the boundary components containing the endpoints of $\alpha$ (possibly $\beta_{1}=\beta_{2}$ ), namely, the boundary geodesics of the corresponding boundary components. If $\beta_{1} \neq \beta_{2}$, then $\alpha$ crosses each of them exactly once; if $\beta_{1}=\beta_{2}$, then $\alpha$ crosses $\beta_{1}$ exactly twice, probably at the same point. Let $l_{S}(\gamma)$ be the length of the geodesic segment of $\alpha$ between $\beta_{1}$ and $\beta_{2}$. We define the modified length spectrum on $T\left(S_{0}\right)$ by

$$
d_{M L}\left(\left[S_{1}, f_{1}\right],\left[S_{2}, f_{2}\right]\right)=\log \sup _{\gamma \in \Sigma_{S_{1}}}\left\{\frac{l_{S_{2}}\left(f_{2} \circ f_{1}^{-1}(\gamma)\right)}{l_{S_{1}}(\gamma)}, \frac{l_{S_{1}}(\gamma)}{l_{S_{2}}\left(f_{2} \circ f_{1}^{-1}(\gamma)\right)}\right\},
$$

where $\Sigma_{S_{0}}=\Sigma_{S_{0}}^{\prime} \cup \Sigma_{S_{0}}^{\prime \prime}$. 
In this paper, we first prove the following:

Theorem 1. Assume that $S_{0}$ is a Riemann surface with boundary. Then the modified length spectrum function $d_{M L}$ defines a metric on $T\left(S_{0}\right)$.

Then we assume that $S_{0}$ is a Riemann surface of type $(g, m, k)$, where $g, m$ and $k$ are the genus, the number of punctures and the number of ideal boundaries, respectively, with $k>0$ and $6 g-6+m+3 k>0$. Under these assumptions, we show the following results.

Theorem 2. The identity map

$$
\text { id: }\left(T\left(S_{0}\right), d_{T}\right) \rightarrow\left(T\left(S_{0}\right), d_{M L}\right)
$$

is continuous, but the inverse map is not.

Corollary 1. The topologies induced by $d_{M L}$ and $d_{T}$ on $T\left(S_{0}\right)$ are not equivalent.

Theorem 3. The metric space $\left(T\left(S_{0}\right), d_{M L}\right)$ is not complete.

\section{Modified length spectrum}

In this section, we prove Theorem 1 . Notice that by using the definition, it is easy to verify that $d_{M L}$ is nonnegative and symmetric, and satisfies the triangle inequality. The main work is to show that it separates points. We first introduce some notation.

(1) Let $L_{x, y}$ denote the geodesic in the unit disk $\mathbf{D}$ or the upper half-plane $\mathbf{H}$ with respect to the hyperbolic metric that connects two points $x$ and $y$ on the boundary of $\mathbf{D}$ or $\mathbf{H}$.

(2) If a geodesic $L$ intersects two geodesics $L_{1}$ and $L_{2}$, then $l\left(L ; L_{1}, L_{2}\right)$ denotes the length, in the hyperbolic metric, of the segment of $L$ between $L_{1}$ and $L_{2}$.

Proposition 1. Let $L_{1}$ and $L_{2}$ be two disjoint geodesics in $\mathbf{H}$ without any common endpoint and let $L_{x_{0}, y_{0}}$ be their common orthogonal. Then $l\left(L_{x_{0}, y} ; L_{1}, L_{2}\right)>$ $l\left(L_{x_{0}, y_{0}} ; L_{1}, L_{2}\right)$ for any geodesic $L_{x_{0}, y}$ crossing $L_{1}$ and $L_{2}$ with $y_{0} \neq y$. Moreover, for any given value $l_{0}>l\left(L_{x_{0}, y_{0}} ; L_{1}, L_{2}\right)$ there exist exactly two geodesics $L_{x_{0}, y_{1}}$ and $L_{x_{0}, y_{2}}$ crossing $L_{1}$ and $L_{2}$ such that $l\left(L_{x_{0}, y_{1}} ; L_{1}, L_{2}\right)=l\left(L_{x_{0}, y_{2}} ; L_{1}, L_{2}\right)=l_{0}$. These geodesics are contained in different connected components of $\mathbf{H} \backslash L_{x_{0}, y_{0}}$.

Proof. Without loss of generality, we may assume that $L_{x_{0}, y_{0}}=L_{0, \infty}, L_{1}=L_{-1,1}$, $L_{2}=L_{-b, b}$ for some $b>1$. Let $c$ be a positive number with $2 c>b$. Then the intersection points of $L_{0,2 c}$ with $L_{1}$ and $L_{2}$ are the solutions of the following systems respectively:

$$
\begin{cases}x^{2}+y^{2} & =1 \\ (x-c)^{2}+y^{2} & =c^{2}\end{cases}
$$

and

$$
\begin{cases}x^{2}+y^{2} & =b^{2}, \\ (x-c)^{2}+y^{2} & =c^{2} .\end{cases}
$$

Solving these systems, we see that the $x$-coordinates of the intersection points of $L_{0,2 c}$ with $L_{1}$ and $L_{2}$ are given by $1 / 2 c$ and $b^{2} / 2 c$ respectively. Now we parameterize the segment of $L_{0,2 c}$ between $L_{1}$ and $L_{2}$ by the equation

$$
\gamma_{0,2 c}(t)=t+i \sqrt{c^{2}-(t-c)^{2}}=t+i \sqrt{2 c t-t^{2}}, t \in\left[1 / 2 c, b^{2} / 2 c\right] .
$$


Then

$$
\begin{aligned}
l\left(L_{0,2 c} ; L_{1}, L_{2}\right) & =\int_{\frac{1}{2 c}}^{\frac{b^{2}}{2 c}} \frac{\left|\gamma_{0,2 c}^{\prime}(t)\right|}{\operatorname{Im}\left(\gamma_{0,2 c}(t)\right)} d t=\int_{\frac{1}{2 c}}^{\frac{b^{2}}{2 c}} \frac{1}{\sqrt{2 c t-t^{2}}} \sqrt{1+\frac{(c-t)^{2}}{2 c t-t^{2}}} d t \\
& =\int_{\frac{1}{2 c}}^{\frac{b^{2}}{2 c}} \frac{c}{(2 c-t) t} d t=\ln b+\frac{1}{2} \ln \frac{4 c^{2}-1}{4 c^{2}-b^{2}}
\end{aligned}
$$

Let

$$
h(c)=l\left(L_{0,2 c} ; L_{1}, L_{2}\right)=\ln b+\frac{1}{2} \ln \frac{4 c^{2}-1}{4 c^{2}-b^{2}} .
$$

The derivative of $h(c)$ is given by

$$
h^{\prime}(c)=\frac{4 c}{4 c^{2}-1}-\frac{4 c}{4 c^{2}-b^{2}} .
$$

Since $2 c>b$ and $b>1, h^{\prime}(c)<0$. It follows that $h$ is a strictly decreasing function of $c$ as soon as $c>b / 2>1 / 2$, which implies that the length decreases as $c$ goes to $\infty$. In fact, $h(\infty)=\ln b=l\left(L_{0, \infty} ; L_{1}, L_{2}\right)$.

Similarly, for $c<0$ with $2 c<-b, g(c)=h(-c)$ is the length of $\gamma_{0,2 c}$. Since $g^{\prime}(c)=-h^{\prime}(-c)>0$, it follows that for $c<-b / 2, g$ is an strictly increasing function of $c$ with $g(-\infty)=\ln b=l\left(L ; L_{0, \infty}, L_{2}\right)$.

Now we prove that $d_{M L}$ separates points. Given any two points $\left[S_{1}, f_{1}\right]$ and $\left[S_{2}, f_{2}\right]$ in $T\left(S_{0}\right)$, we use the same symbols to denote their equivalent classes in $T^{R}\left(S_{0}\right)$. Then

$$
d_{L}\left(\left[S_{1}, f_{1}\right],\left[S_{2}, f_{2}\right]\right) \leq d_{M L}\left(\left[S_{1}, f_{1}\right],\left[S_{2}, f_{2}\right]\right) .
$$

Suppose that $d_{M L}\left(\left[S_{1}, f_{1}\right],\left[S_{2}, f_{2}\right]\right)=0$. Then $d_{L}\left(\left[S_{1}, f_{1}\right],\left[S_{2}, f_{2}\right]\right)=0$. Since $d_{L}$ is a metric in $T^{R}\left(S_{0}\right)$, it follows that $f_{2} \circ f_{1}^{-1}$ is homotopic to a conformal mapping $c: S_{1} \rightarrow S_{2}$. We need to prove that $f=f_{2} \circ f_{1}^{-1}$ is homotopic to $c$ relative to boundary.

The following is a classical theorem which can be found in [4].

Theorem 4. Let $S_{1}$ and $S_{2}$ be two hyperbolic Riemann surfaces and let $f_{i}: S_{1} \rightarrow$ $S_{2}, i=1,2$, be two quasiconformal mappings. Assume that the Fuchsian group $G_{1}$ representing $S_{1}$ is non-elementary. Then

(1) $f_{1}$ is homotopic to $f_{2}$ if and only if they can be lifted to mappings of $\mathbf{H}$ which agree on the limit set of $G_{1}$; and

(2) $f_{1}$ is homotopic to $f_{2}$ relative to boundary if and only if they can be lifted to mappings of $\mathbf{H}$ which agree on $\widehat{\mathbf{R}}$.

Given a Riemann surface $S$ with boundary, let $G$ be the group uniformizing $S$. The universal covering $\pi: \mathbf{D} \rightarrow \operatorname{int}(S)$ extends to a covering $\pi: \overline{\mathbf{D}} \backslash \Lambda(G) \rightarrow S$, where $\Lambda(G)$ is the limiting set of $G$. Then a quasiconformal mapping $f$ from $S$ to $S$ lifts to a mapping $F: \overline{\mathbf{D}} \backslash \Lambda(G) \rightarrow \overline{\mathbf{D}} \backslash \Lambda(G)$, which extends to a homeomorphism of $\overline{\mathbf{D}}$. In this paper, in order to avoid repeating the details of the maps $\pi$ and $F$ on the boundary of $\mathbf{D}$, we say in brief that $\pi: \mathbf{D} \rightarrow S$ is a universal covering map for $S$ and $F: \mathbf{D} \rightarrow \mathbf{D}$ is a lifting of $f$.

We continue to show that $d_{M L}$ separates points in $T\left(S_{0}\right)$. Following the notation introduced in this section and using the previous theorem, we let $F: \mathbf{H} \rightarrow \mathbf{H}$ and $C: \mathbf{H} \rightarrow \mathbf{H}$ be liftings of $f$ and $c$ respectively that agree on the limit set $\Lambda$ of the Fuchsian group $G_{1}$ uniformazing $S_{1}$. Clearly, $F \circ C^{-1}$ maps each connected component 
of $\mathbf{S}^{1} \backslash \Lambda$ onto itself. It remains to show $F \circ C^{-1}$ is the identity on each connected component. Let $L$ be a lifting of a boundary geodesic $\beta$ of $S_{1}$. Then one of the arcs bounded by the endpoints of $L$ on $\mathbf{S}^{1}$ is a connected component of $\mathbf{S}^{1} \backslash \Lambda$ and each connected component is bounded by the endpoints of a lifting of a boundary geodesic of $S_{1}$.

Let $L_{1}$ and $L_{2}$ be two different liftings of a boundary geodesic $\beta$ of $S_{1}$, and let $L_{x_{0}, y_{0}}$ be their common perpendicular. The mapping $C$, being conformal, maps $L_{x_{0}, y_{0}}$ to the common perpendicular between $C\left(L_{1}\right)$ and $C\left(L_{2}\right)$. By assumption, $l_{S_{2}}(f(\gamma))=l_{S_{1}}(\gamma)$ for every $\gamma \in \Sigma_{S_{0}}^{\prime \prime}$. On the other hand, since $c$ is an isometry, we must have $l_{S_{1}}(\gamma)=l_{S_{2}}(c(\gamma))$ for every $\gamma \in \Sigma_{S_{0}}^{\prime \prime}$. It follows that

$$
l\left(L_{F\left(x_{0}\right), F\left(y_{0}\right)} ; C\left(L_{1}\right), C\left(L_{2}\right)\right)=l\left(L_{x_{0}, y_{0}} ; L_{1}, L_{2}\right)=l\left(L_{C\left(x_{0}\right), C\left(y_{0}\right)} ; C\left(L_{1}\right), C\left(L_{2}\right)\right) .
$$

Since the common perpendicular segment is the unique segment of the smallest length among all segments connecting two geodesics, it follows that $F\left(x_{0}\right)=C\left(x_{0}\right)$ and $F\left(y_{0}\right)=C\left(y_{0}\right)$.

Let $I_{i}$ be the interval on the real line bounded by the endpoints of $L_{i}$. It projects to the component of the ideal boundary of $S_{0}$ homotopic to $\beta$. Assume $x_{0} \in I_{1}$ and $y_{0} \in I_{2}$. For any point $x \in I_{2} \backslash\left\{y_{0}\right\}$, consider the geodesic $L_{x_{0}, x}$. Then

$$
l\left(L_{F\left(x_{0}\right), F(x)} ; C\left(L_{1}\right), C\left(L_{2}\right)\right)=l\left(L_{x_{0}, x} ; L_{1}, L_{2}\right)=l\left(L_{C\left(x_{0}\right), C(x)} ; C\left(L_{1}\right), C\left(L_{2}\right)\right) .
$$

It follows from Proposition 1 that $F(x)=C(x)$. This argument can be applied to any point $x \in \widehat{\mathbf{R}}$ that is not in the limit set $\Lambda$ of $G_{1}$. Thus both maps agree on the whole boundary of $\mathbf{H}$. It follows from Theorem 4 that their projections to the surfaces are homotopic to each other modulo boundary. Thus, $d_{M L}$ separates points in $T\left(S_{0}\right)$ and then Theorem 1 follows.

\section{Proofs of main results}

The following is a well known result due to Wolpert (see [1]).

Lemma 1. Let $S_{1}$ and $S_{2}$ be two homeomorphic hyperbolic Riemann surfaces. If $f: S_{1} \rightarrow S_{2}$ is a quasiconformal mapping, then

$$
K(f) \geq \frac{l_{S_{2}}(f(\gamma))}{l_{S_{1}}(\gamma)} \text { for any } \gamma \in \Sigma_{S_{1}}^{\prime} .
$$

As an immediate consequence we obtain that for any two points $\tau_{1}, \tau_{2} \in T^{R}\left(S_{0}\right)$, $d_{L}\left(\tau_{1}, \tau_{2}\right) \leq d_{T}\left(\tau_{1}, \tau_{2}\right)$. It follows that the identity map

$$
\text { id: }\left(T^{R}\left(S_{0}\right), d_{T}\right) \rightarrow\left(T^{R}\left(S_{0}\right), d_{L}\right)
$$

is continuous. Theorem 2 is an analogy of the previous statement to $T\left(S_{0}\right)$ under the Teichmüller metric $d_{T}$ and the modified length spectrum $d_{M L}$. Before proving this theorem, we introduce some lemmas.

Lemma 2. Let $L_{a, b}, a<b$, be a geodesic in $\mathbf{H}$ and $I$ a closed interval contained in $(a, b)$. Assume that $\left\{L_{a_{n}, b_{n}}\right\}, b<a_{n}<b_{n}$, is a sequence of geodesics in $\mathbf{H}$ such that the hyperbolic distance between $L_{a_{n}, b_{n}}$ and $L_{a, b}$ is bounded below by some $\epsilon>0$ for every $n$. Then

$$
\inf _{x \in I, y_{n} \in\left(a_{n}, b_{n}\right)} l\left(L_{x, y_{n}} ; L_{a, b}, L_{a_{n}, b_{n}}\right) \rightarrow \infty \text { as } n \rightarrow \infty
$$

provided that $a_{n}, b_{n} \rightarrow b$ as $n \rightarrow \infty$. 
Proof. For each $n$, we use the Möbius transformation

$$
T_{n}(z)=\frac{z-b}{z-a} \frac{b_{n}-a}{b_{n}-b}
$$

to map $L_{a, b}$ and $L_{a_{n}, b_{n}}$ to $L_{\infty, 0}$ and $L_{T_{n}\left(a_{n}\right), 1}$ respectively. By the assumptions, the distance between $L_{\infty, 0}$ and $L_{T_{n}\left(a_{n}\right), 1}$ is bounded below by some $\epsilon>0$. Then there exists $r>0$ such that $T_{n}\left(a_{n}\right) \geq r$ for every $n$. For any $x \in I$ and $y_{n} \in\left(a_{n}, b_{n}\right)$, we have

$$
l\left(L_{x, y_{n}} ; L_{a, b}, L_{a_{n}, b_{n}}\right)=l\left(L_{T_{n}(x), T_{n}\left(y_{n}\right)} ; L_{\infty, 0}, L_{T_{n}\left(a_{n}\right), 1}\right) \geq l\left(L_{T_{n}(x), T_{n}\left(y_{n}\right)} ; L_{\infty, 0}, L_{r, 1}\right) .
$$

Since $T_{n}(x) \rightarrow-\infty$ uniformly for $x \in I$ as $n \rightarrow \infty$, it follows that

$$
\inf _{x \in I, y_{n} \in\left(a_{n}, b_{n}\right)} l\left(L_{T_{n}(x), T_{n}\left(y_{n}\right)} ; L_{\infty, 0}, L_{r, 1}\right) \rightarrow \infty
$$

as $n \rightarrow \infty$.

Let $x$ and $y$ be two distinct points on the unit circle $\mathbf{S}^{1}$. Denote by $\lceil x, y\rceil$ the circular arc on $\mathbf{S}^{1}$ connecting $x$ to $y$ in counterclockwise direction.

For any $d>0$, choose $b=b(d) \in \mathbf{S}^{1}$ such that $0<\arg (b)<\pi / 2$ and the hyperbolic distance between $L_{-i, i}$ and $L_{\bar{b}, b}$ is $d$. We call $L_{\bar{b}, b}$ the $d$-standard geodesic. Now we assume that a positive number $s$ is sufficiently small (depending on $b(d)$ ). Let $I_{s}=\left\lceil x_{s}, y_{s}\right\rceil \subseteq\lceil i,-i\rceil$ and $J_{s, b}=\left\lceil z_{s}, w_{s}\right\rceil \subseteq\lceil\bar{b}, b\rceil$ be the arcs on $\mathbf{S}^{1}$ such that the length of each of the arcs $\left\lceil i, x_{s}\right\rceil,\left\lceil y_{s},-i\right\rceil,\left\lceil\bar{b}, z_{s}\right\rceil$, and $\left\lceil w_{s}, b\right\rceil$ is equal to $s$.

Lemma 3. Assume that $0<d_{0}<d_{1}$. For any $D>d_{1}$, there exists $s_{0}>0$ such that for any $d_{0} \leq d \leq d_{1}$, if $l\left(L_{p, q} ; L_{-i, i}, L_{\overline{b(d)}, b(d)}\right) \leq D$, then $p \in I_{s_{0}}$ and $q \in J_{s_{0}, b(d)}$.

Proof. Let $D>d_{1}$. For any $d \in\left[d_{0}, d_{1}\right]$, there exists a maximal $s$, denoted by $s(d)$, such that if $l\left(L_{p, q} ; L_{-i, i}, L_{\overline{b(d)}, b(d)}\right) \leq D$ then $p \in I_{s}$ and $q \in J_{s, b(d)}$. The function $d \mapsto s(d)$ is a continuous function defined on the compact interval $\left[d_{0}, d_{1}\right]$. Then $s_{0}=\min _{d \in\left[d_{0}, d_{1}\right]} s(d)$ satisfies the conclusion of the lemma.

Lemma 4. Assume that $0<d_{0}<d_{1}$ and also assume that $s_{0}$ is a positive number small enough (only depending on $d_{1}$ ). Then for any $\epsilon>0$, there exists $\delta>0$ depending on $d_{0}, d_{1}, s_{0}$ and $\epsilon$ such that

(1) for every $d \in\left[d_{0}, d_{1}\right]$,

(2) for every $x, y, z, w \in \mathbf{S}^{1}$,

(3) for every $p_{1}, p_{2} \in I_{s_{0}}$, and

(4) for every $q_{1}, q_{2} \in J_{s_{0}, b(d)}$,

if each of the numbers $\left|p_{1}-p_{2}\right|,\left|q_{1}-q_{2}\right|,|x-i|,|y+i|,|z-\overline{b(d)}|$ and $|w-b(d)|$ is less than $\delta$, then

$$
\left|\log \frac{l\left(L_{p_{1}, q_{1}} ; L_{-i, i}, L_{\overline{b(d)}, b(d)}\right)}{l\left(L_{p_{2}, q_{2}} ; L_{x, y}, L_{z, w}\right)}\right|<\epsilon .
$$

Proof. Suppose the lemma is false. Then there exists $\epsilon>0$ such that for every $\delta_{n}=1 / n$, there exist

(1) $d_{n} \in\left[d_{0}, d_{1}\right]$

(2) $x_{n}, y_{n}, z_{n}, w_{n} \in \mathbf{S}^{1}$ with $\left|x_{n}-i\right|,\left|y_{n}+i\right|,\left|z_{n}-\overline{b\left(d_{n}\right)}\right|$ and $\left|w-b\left(d_{n}\right)\right|<1 / n$,

(3) $p_{1}^{(n)}, p_{2}^{(n)} \in I_{s_{0}}$ with $\left|p_{1}^{(n)}-p_{2}^{(n)}\right|<1 / n$, and

(4) $q_{1}^{(n)}, q_{2}^{(n)} \in J_{s_{0}, b\left(d_{n}\right)}$ with $\left|q_{1}^{(n)}-q_{2}^{(n)}\right|<1 / n$ 
such that

$$
\left|\log \frac{l\left(L_{p_{1}^{(n)}, q_{1}^{(n)}} ; L_{-i, i}, L_{\overline{b\left(d_{n}\right)}, b\left(d_{n}\right)}\right)}{l\left(L_{p_{2}^{(n)}, q_{2}^{(n)}} ; L_{x_{n}, y_{n}}, L_{z_{n}, w_{n}}\right)}\right| \geq \epsilon .
$$

We may assume, by passing to subsequences, that

(1) $d_{n} \rightarrow d^{(0)}$, which implies $b\left(d_{n}\right) \rightarrow b\left(d^{(0)}\right)$;

(2) $x_{n} \rightarrow i, y_{n} \rightarrow-i, z_{n} \rightarrow b\left(d^{(0)}\right), w_{n} \rightarrow \overline{b\left(d^{(0)}\right)}$; and

(3) $p_{1}^{(n)} \rightarrow p_{1}^{(0)}, q_{1}^{(n)} \rightarrow q_{1}^{(0)}$ and thus $p_{2}^{(n)} \rightarrow p_{1}^{(0)}, q_{2}^{(n)} \rightarrow q_{1}^{(0)}$.

Since $p_{1}^{(n)} \in I_{s_{0}}$ and $q_{1}^{(n)} \in J_{s_{0}, b\left(d_{n}\right)}$, it follows that $p_{1}^{(0)}$ and $q_{1}^{(0)}$ are contained in the interior of $\lceil i,-i\rceil$ and $\left\lceil\overline{b\left(d^{(0)}\right)}, b\left(d^{(0)}\right)\right\rceil$ respectively. Then we can choose $n$ sufficiently large so that

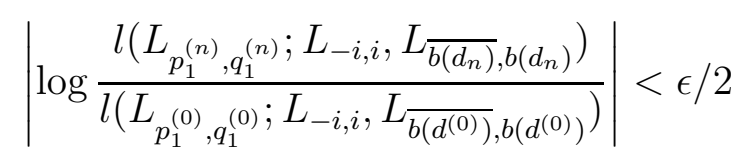

and

$$
\left|\log \frac{l\left(L_{p_{2}^{(n)}, q_{2}^{(n)}} ; L_{x_{n}, y_{n}}, L_{z_{n}, w_{n}}\right)}{l\left(L_{p_{1}^{(0)}, q_{1}^{(0)}} ; L_{-i, i}, L_{\overline{b\left(d^{(0)}\right)}, b\left(d^{(0)}\right)}\right)}\right|<\epsilon / 2 .
$$

Combining both inequalities, we obtain

$$
\left|\log \frac{l\left(L_{p_{1}^{(n)}, q_{1}^{(n)}} ; L_{-i, i}, L_{\overline{b\left(d_{n}\right)}, b\left(d_{n}\right)}\right)}{l\left(L_{p_{2}^{(n)}, q_{2}^{(n)}} ; L_{x_{n}, y_{n}}, L_{z_{n}, w_{n}}\right)}\right|<\epsilon
$$

for $n$ sufficiently large. This is a contradiction to the inequality (3.1).

For the rest of this paper, we consider Riemann surfaces $S$ of type $(g, m, k)$, where $g, m$ and $k$ are the genus, the number of punctures and the number of ideal boundaries, respectively, with $k>0$ and $6 g-6+m+3 k>0$. Let $S^{d}$ be the double of $S$. Then $S^{d}$ is of type $(2 g+k-1,2 m, 0)$ and the boundary curves of $S$ become closed geodesics on $S^{d}$. The intrinsic metric on $S$ is defined to be the restriction to $S$ of the hyperbolic metric on $S^{d}$. The Nielsen kernel $\widetilde{S}$ of $S$ is the Riemann surface of the same type obtained by removing from $S$ the $k$ funnels formed by the boundary geodesics and the ideal boundary of $S$. The surface $S$ is called the Nielsen extension of $\widetilde{S}$ and one of them is uniquely determined by the other. For more details about the Nielsen kernel of a Riemann surface, the reader is referred to [2].

Bers [2] proved the following result.

Lemma 5. The intrinsic metric on $\widetilde{S}$ is equal to the restriction to $\widetilde{S}$ of the hyperbolic metric on $S$.

Before we prove our theorems, we prepare a couple of more lemmas.

Lemma 6. For any curve $\gamma \in \Sigma_{S}^{\prime \prime}$,

$$
\frac{l_{\widetilde{S}^{d}}\left(\widetilde{\gamma}^{d}\right)}{2} \leq l_{S}(\gamma) \leq \frac{l_{\widetilde{S}^{d}}\left(\widetilde{\gamma}^{d}\right)}{2}+2 M
$$

where $\widetilde{\gamma}$ is the restriction of $\gamma$ to $\widetilde{S}, \widetilde{\gamma}^{d}$ is the double of $\widetilde{\gamma}$, and

$$
M=\max \left\{l_{S}(\beta): \beta \text { is a boundary geodesic in } S\right\} .
$$


Proof. For any curve $\alpha$ in $\widetilde{S}^{d}$, let $\widetilde{l}_{\widetilde{S}^{d}}(\alpha)$ denote the length of the curve $\alpha$ in the hyperbolic metric on $\widetilde{S}^{d}$. Let $\gamma$ be an arc in $\Sigma_{S}^{\prime \prime}$; without loss of generality we may assume that it is a geodesic arc. By Lemma $5, \widetilde{l}_{\widetilde{S}^{d}}(\widetilde{\gamma})=l_{S}(\gamma)$. Since $\widetilde{l}_{\widetilde{S}^{d}}(\widetilde{\gamma})=\widetilde{l}_{\widetilde{S}^{d}}\left(\widetilde{\gamma}^{d}\right) / 2 \geq l_{\widetilde{S}^{d}}\left(\widetilde{\gamma}^{d}\right) / 2$, the left-hand side inequality follows.

Recall that the geodesic arc $\gamma$ either crosses two distinct boundary geodesics exactly once or one exactly twice. Let $\beta_{1}, \beta_{2} \in \Sigma_{S}^{\prime}$ be the ones crossed by $\gamma$ at the points $p_{1}$ and $p_{2}$ respectively. If $\beta_{1}=\beta_{2}$, then $p_{1}$ and $p_{2}$ belong to the same geodesic boundary and in this case $p_{1}$ may be equal to $p_{2}$. Let $\beta$ be the closed geodesic on $\widetilde{S}^{d}$ in the homotopy class of $\widetilde{\gamma}^{d}$. Then $\beta$ crosses $\beta_{1}$ and $\beta_{2}$ in a similar fashion as $\gamma$. Denote these two crossing points by $q_{1}$ and $q_{2}$ respectively. Let $\beta_{i}^{\prime}$ be one of the two segments of $\beta_{i}$ joining $p_{i}$ to $q_{i}, i=1,2$. Then

$$
\begin{aligned}
l_{S}(\gamma) & =\widetilde{l}_{\widetilde{S}^{d}}(\widetilde{\gamma}) \leq \widetilde{l}_{\widetilde{S}^{d}}\left(\beta_{1}^{\prime}\right)+\widetilde{l}_{\widetilde{S}^{d}}(\beta \cap \widetilde{S})+\widetilde{l}_{\widetilde{S}^{d}}\left(\beta_{2}^{\prime}\right)=\widetilde{l}_{\widetilde{S}^{d}}\left(\beta_{1}^{\prime}\right)+\frac{1}{2} \widetilde{l}_{\widetilde{S}^{d}}(\beta)+\widetilde{l}_{\widetilde{S}^{d}}\left(\beta_{2}^{\prime}\right) \\
& \leq \widetilde{S}^{d}\left(\beta_{1}\right)+\frac{1}{2} \widetilde{l}_{\widetilde{S}^{d}}(\beta)+l_{\widetilde{S}^{d}}\left(\beta_{2}\right) \leq \frac{1}{2} \widetilde{l}_{\widetilde{S}^{d}}(\beta)+2 M=\frac{1}{2} l_{\widetilde{S}^{d}}\left(\widetilde{\gamma}^{d}\right)+2 M
\end{aligned}
$$

where the second equality follows from the fact that $\beta$ is homotopic to $\widetilde{\gamma}^{d}$, which is symmetric with respect to the geodesics on $\tilde{S}^{d}$ coming from the boundary geodesics of $S$. The right-hand inequality of (3.2) now follows.

Lemma 7. Let $f_{n}: S_{0} \rightarrow S_{n}, n=1,2, \cdots$, be a sequence of quasiconformal mappings such that $K\left(f_{n}\right) \rightarrow 1$ as $n \rightarrow \infty$. Then there exists a sequence of quasiconformal mappings $h_{n}: S_{0} \rightarrow S_{n}$ such that (i) if $n$ is sufficiently large, then $h_{n}$ is homotopic to $f_{n}$ relative to boundary and $h_{n}$ preserves the Nielsen kernel of $S_{n}$, i.e., $h_{n}\left(\widetilde{S}_{0}\right)=\widetilde{S}_{n}$, and (ii) $K\left(h_{n}\right) \rightarrow 1$ as $n \rightarrow \infty$.

Proof. For each $n=0,1,2, \cdots$, let $G_{n}$ be the group uniformizing $S_{n}$, let $\pi_{n}: \mathbf{D} \rightarrow$ $S_{n}$ be the universal covering map, and let $F_{n}: \mathbf{D} \rightarrow \mathbf{D}$ be a lifting of $f_{n}$ normalized to fix three points. Then $K\left(F_{n}\right) \rightarrow 1$ and $F_{n}$ converges uniformly to the identity map.

Let $D_{0} \subseteq \mathbf{D}$ be a Dirichlet fundamental domain for $G_{0}$. Then $F_{n}\left(D_{0}\right)$ is a fundamental domain for $G_{n}$. From now on, we assume that $n$ is sufficiently large. Then $F_{n}$ is very close to the identity map. It follows that the vertices of $D_{0}$ are moved very little by $F_{n}$. Then for each edge $e$ of $D_{0}$, replace $F_{n}(e)$ by the geodesic segment connecting the endpoints of $F_{n}(e)$. These new edges bound a domain $D_{n}$, which is a new fundamental domain for $G_{n}$. We briefly explain why it is so in two steps.

Note that when $n$ is sufficiently large, nonadjacent edges of $D_{n}$ do not intersect.

Step 1: We show that each orbit under the action of $G_{n}$ intersects $D_{n}$. Let $p \in \mathbf{D}$. Then there exists $g \in G_{n}$ such that $g(p) \in F_{n}\left(D_{0}\right)$. Suppose $g(p) \notin D_{n}$. Then $g(p)$ belongs to a connected component of $F_{n}\left(D_{0}\right) \backslash D_{n}$. This connected component is bounded by a segment of an edge (or probably a full edge) of $D_{n}$ and a segment of $F_{n}(e)$ (or probably the full curve $F_{n}(e)$ ), where $e$ is an edge of $D_{0}$. The curve $F_{n}(e)$ is paired to another curve $F_{n}\left(e^{\prime}\right)$ by an element $g_{2} \in G_{n}$, where $e^{\prime}$ is an edge of $D_{0}$. Then $\left(g_{2} \circ g\right)(p)$ is contained in $D_{n} \backslash F_{n}\left(D_{0}\right)$. Thus, $\left(g_{2} \circ g\right)(p) \in D_{n}$.

Step 2: We prove that the interior $\operatorname{int}\left(D_{n}\right)$ of $D_{n}$ contains at most one point from each orbit under $G_{n}$. Suppose that there are two points $p_{1}$ and $p_{2}$ of $\operatorname{int}\left(D_{n}\right)$ that lie on the same orbit. Assume that $p_{1} \in \operatorname{int}\left(D_{n}\right) \backslash F_{n}\left(D_{0}\right)$. Using an argument similar to the one in Step 1, we can show that there exists $g_{1} \in G_{n}$ such that 
$g_{1}\left(p_{1}\right)$ belongs to the interior of $F_{n}\left(D_{0}\right)$. Clearly, there exists $g_{2} \in G_{n}$ such that $g_{2}\left(p_{2}\right) \in F_{n}\left(D_{0}\right)$. The positions of $g_{1}\left(p_{1}\right)$ and $g_{2}\left(p_{2}\right)$ make a contradiction since they stay on the same orbit. Therefore, $p_{1} \in F_{n}\left(D_{0}\right)$. With the same reasoning, we know $p_{2} \in F_{n}\left(D_{0}\right)$. Since $F_{n}\left(D_{0}\right)$ is a fundamental domain for $G_{n}$, it follows that there exist two edges $e_{1}$ and $e_{2}$ of $D_{0}$ and an element $g \in G_{n}$ such that $p_{1} \in F_{n}\left(e_{1}\right)$, $p_{2} \in F_{n}\left(e_{2}\right), g\left(F_{n}\left(e_{1}\right)\right)=F_{n}\left(e_{2}\right)$ and $g\left(p_{1}\right)=p_{2}$. All these force $p_{1}$ and $p_{2}$ to stay on a pair of edges of $D_{n}$; otherwise, one of them belongs to $\operatorname{int}\left(D_{n}\right)$ and the other has to be outside of $D_{n}$. Both situations contradict the assumption. Therefore, $\operatorname{int}\left(D_{n}\right)$ contains at most one point from each orbit.

Now for each such large $n$, let $\widetilde{D}_{n}=\pi_{n}^{-1}\left(\widetilde{S}_{n}\right) \bigcap D_{n}$. The region $\widetilde{D}_{n}$ is a convex polygon whose vertices are either in $\mathbf{D}$ or on $\partial \mathbf{D}$ and it projects to the Nielsen kernel $\widetilde{S}_{n}$ of $S_{n}$. Each $D_{n}$ is the convex hull of its vertices, thus we can construct a piecewise hyperbolic affine map $H_{n}: \widetilde{D}_{0} \rightarrow \widetilde{D}_{n}$ mapping vertices to vertices. In order to extend $H_{n}$ to $D_{0}$, we foliate each connected component of $D_{0} \backslash \widetilde{D}_{0}$ by geodesic rays starting at $\partial \widetilde{D}_{0}$ and ending at $\partial \mathbf{D} \cap \bar{D}_{0}$, where $\bar{D}_{0}$ is the closure of $D_{0}$ in the closed unit disk with respect to the Euclidean metric. For each such geodesic ray with endpoints $z \in \partial \widetilde{D}_{0}$ and $x \in \partial \mathbf{D} \cap \bar{D}_{0}$, we let $H_{n}$ map this ray onto the geodesic ray starting at $H_{n}(z)$ and ending at $F_{n}(x)$ such that the distances from the starting points are preserved. Finally we extend $H_{n}$ to the whole hyperbolic disk by using the actions of $G_{0}$ and $G_{n}$ on $\mathbf{D}$. By Theorem $4, H_{n}$ projects to a mapping $h_{n}: S_{0} \rightarrow S_{n}$ which is homotopic to $f_{n}$ relative to boundary and by the construction $h_{n}\left(\widetilde{S}_{0}\right)=\widetilde{S}_{n}$. Moreover, since $F_{n}$ converges uniformly to the identity, the vertices of $D_{n}$ approach the vertices of $D_{0}$ as $n \rightarrow \infty$. Thus, $H_{n} \rightarrow$ id and $K\left(H_{n}\right) \rightarrow 1$ as $n \rightarrow \infty$. Hence $K\left(h_{n}\right) \rightarrow 1$ as $n \rightarrow \infty$.

Theorem 5. The identity function id: $\left(T\left(S_{0}\right), d_{T}\right) \rightarrow\left(T\left(S_{0}\right), d_{M L}\right)$ is continuous.

Proof. Let $\left\{\tau_{n}\right\}$ be a sequence of points in $T\left(S_{0}\right)$ converging to a point $\tau$ in the Teichmüller metric and let $\epsilon>0$ be given. Without loss of generality, we may assume that $\tau=\left[S_{0}\right.$,id]. Let $\tau_{n}=\left[S_{n}, f_{n}\right]$, by Lemma 7 , we may assume that $f_{n}\left(\widetilde{S}_{0}\right)=\widetilde{S}_{n}$ and $K\left(f_{n}\right) \rightarrow 1$ as $n \rightarrow \infty$. Consider $\left[S_{n}, f_{n}\right]$ and $\left[S_{0}\right.$, id] as elements of $T^{R}\left(S_{0}\right)$. As we mention in the introduction, it is proved in [7] that $d_{L}$ and $d_{T}$ are topologically equivalent in $T^{R}\left(S_{0}\right)$. Thus, there exists $N_{1}$ such that

$$
\left|\log \frac{l_{S_{n}}\left(f_{n}(\gamma)\right)}{l_{S_{0}}(\gamma)}\right|<\epsilon
$$

for every $n>N_{1}$ and every $\gamma \in \Sigma_{S_{0}}^{\prime}$. For each $n=0,1,2, \cdots$, let

$$
M_{n}=\max \left\{l_{S_{n}}(\beta): \beta \text { is a boundary geodesic in } S_{n}\right\} \text {. }
$$

Then the previous property (3.3) implies that $M_{n}$ converges to $M_{0}$ as $n \rightarrow \infty$. Hence there exists a constant $M^{\prime}>0$ such that $M_{n} \leq M^{\prime}$ for every $n$.

Let $\gamma \in \Sigma_{S_{0}}^{\prime \prime}$. Since $f_{n}$ maps $\widetilde{S}_{0}$ to $\widetilde{S}_{n}$, it follows that $\widetilde{f_{n}(\gamma)}=\widetilde{f}_{n}(\widetilde{\gamma})$ and $\left(\widetilde{f}_{n}(\widetilde{\gamma})\right)^{d}=$ $\widetilde{f}_{n}^{d}\left(\widetilde{\gamma}^{d}\right)$, where $\widetilde{f}_{n}=\left.f\right|_{\widetilde{S}_{0}}$ and $\widetilde{f}_{n}^{d}: \widetilde{S}_{0}^{d} \rightarrow \widetilde{S}_{n}^{d}$ is the double mapping of $\widetilde{f}_{n}$. Then by lemma 6 and the fact that $M_{n} \leq M^{\prime}$ for any $n=0,1,2, \cdots$, we conclude that

$$
\frac{l_{\widetilde{S}_{0}^{d}}\left(\widetilde{\gamma}^{d}\right)}{2} \leq l_{S_{0}}(\gamma) \leq \frac{l_{\widetilde{S}_{0}^{d}}\left(\widetilde{\gamma}^{d}\right)}{2}+2 M^{\prime}
$$


and

$$
\frac{l_{\widetilde{S}_{n}^{d}}\left(\widetilde{f}_{n}^{d}\left(\widetilde{\gamma}^{d}\right)\right)}{2} \leq l_{S_{n}}\left(f_{n}(\gamma)\right) \leq \frac{l_{\widetilde{S}_{n}^{d}}\left(\widetilde{f}_{n}^{d}\left(\widetilde{\gamma}^{d}\right)\right)}{2}+2 M^{\prime} .
$$

Combining inequalities (3.4) and (3.5), we obtain

or

$$
\frac{\frac{1}{2} l_{\widetilde{S}_{n}^{d}}\left(\widetilde{f}_{n}^{d}\left(\widetilde{\gamma}^{d}\right)\right)}{\frac{1}{2} l_{\widetilde{S}_{0}^{d}}\left(\widetilde{\gamma}^{d}\right)+2 M^{\prime}} \leq \frac{l_{S_{n}}\left(f_{n}(\gamma)\right)}{l_{S_{0}}(\gamma)} \leq \frac{\frac{1}{2} l_{\widetilde{S}_{n}^{d}}\left(\widetilde{f}_{n}^{d}\left(\widetilde{\gamma}^{d}\right)\right)+2 M^{\prime}}{\frac{1}{2} l_{\widetilde{S}_{0}^{d}}\left(\widetilde{\gamma}^{d}\right)}
$$

$$
\frac{\frac{l_{\widetilde{S}_{n}^{d}\left(\widetilde{f}_{n}^{d}\left(\widetilde{\gamma}^{d}\right)\right)}}{l_{\widetilde{S}_{0}^{d}}^{d}\left(\widetilde{\gamma}^{d}\right)}}{1+\frac{4 M^{\prime}}{\left.l_{\widetilde{S}_{0}^{d}} \widetilde{\gamma}^{d}\right)}} \leq \frac{l_{S_{n}}\left(f_{n}(\gamma)\right)}{l_{S_{0}}(\gamma)} \leq \frac{l_{\widetilde{S}_{n}^{d}}\left(\widetilde{f}_{n}^{d}\left(\widetilde{\gamma}^{d}\right)\right)}{l_{\widetilde{S}_{0}^{d}}\left(\widetilde{\gamma}^{d}\right)}+\frac{4 M^{\prime}}{l_{\widetilde{S}_{0}^{d}}\left(\widetilde{\gamma}^{d}\right)}
$$

Lemma 1 and the fact that $K\left(\widetilde{f}_{n}^{d}\right)=K\left(\widetilde{f}_{n}\right) \leq K\left(f_{n}\right)$ imply

$$
\frac{1}{K\left(f_{n}\right)} \leq \frac{l_{\widetilde{S}_{n}^{d}}\left(\widetilde{f}_{n}^{d}\left(\widetilde{\gamma}^{d}\right)\right)}{l_{\widetilde{S}_{0}^{d}}\left(\widetilde{\gamma}^{d}\right)} \leq K\left(f_{n}\right) .
$$

Thus we can choose $D$ and $N_{2}$ sufficiently large such that if $n>N_{2}$ and $l_{S_{0}}(\gamma)>D$, then $l_{\widetilde{S}_{n}^{d}}\left(\widetilde{f}_{n}^{d}\left(\widetilde{\gamma}^{d}\right)\right) / l_{\widetilde{S}_{0}^{d}}\left(\widetilde{\gamma}^{d}\right)$ is sufficiently close to 1 and $4 M^{\prime} / l_{\widetilde{S}_{0}^{d}}\left(\widetilde{\gamma}^{d}\right)$ is sufficiently small. More precisely, we choose $D$ and $N_{2}$ large enough such that

$$
\left|\log \frac{l_{S_{n}}\left(f_{n}(\gamma)\right)}{l_{S_{0}}(\gamma)}\right|<\epsilon
$$

for every $n>N_{2}$ and every $\gamma \in \Sigma_{S_{0}}^{\prime \prime}, l_{S_{0}}(\gamma)>D$. It remains to consider all arcs $\gamma \in \Sigma_{S_{0}}^{\prime \prime}$ with $l_{S_{0}}(\gamma) \leq D$.

Let $G_{0}$ be the Fuchsian group uniformizing $S_{0}$ and let $\pi: \mathbf{D} \rightarrow S_{0}$ be the universal covering map. Let $B$ be a boundary component of $S_{0}$ and $\beta$ the corresponding boundary geodesic. Assume that $\beta^{*}$ is a lifting of $\beta$ in $\mathbf{D}$. Then $\beta^{*}$ separates the unit circle $\mathbf{S}^{1}$ into two open circular arcs and one of them is a cover of $B$ under $\pi$. We denote it by $B^{*}$. Let $I$ be a closed segment of $B^{*}$ such that $I$ minus one of its endpoint covers $B$ exactly once. Without loss of generality, we assume that all elements in $\Sigma_{S_{0}}^{\prime \prime}$ are geodesic arcs. Let $\mathcal{F}$ be the collection of the liftings $\gamma^{*}$ of the elements $\gamma \in \Sigma_{S_{0}}^{\prime \prime}$ with $l_{S_{0}}(\gamma) \leq D$ such that they have one endpoint on $I$. We claim that besides $\beta^{*}$, there are only finitely many lifting geodesics of the boundary geodesics of $S_{0}$ such that they intersect at least one $\gamma^{*} \in \mathcal{F}$. Let $\beta_{1}^{*}, \beta_{2}^{*}, \cdots, \beta_{r}^{*}$ be such lifting geodesics (different from $\beta^{*}$ ).

We show the claim. Suppose there are infinitely many such lifting geodesics $\left\{\beta_{j}^{*}\right\}_{j=1}^{\infty}$ besides $\beta^{*}$. For each $j$, let $\gamma_{j}^{*} \in \mathcal{F}$ such that $\gamma_{j}^{*} \cap \beta_{j}^{*} \neq \emptyset$. If there is a subsequence $\left\{\beta_{j_{k}}^{*}\right\}$ that does not accumulate at one of the endpoints of $\beta^{*}$, then it is easy to see that $l\left(\gamma_{j_{k}}^{*} ; \beta^{*}, \beta_{j_{k}}^{*}\right) \rightarrow \infty$ as $k \rightarrow \infty$, which contradicts the fact that $l\left(\gamma_{j_{k}}^{*} ; \beta^{*}, \beta_{j_{k}}^{*}\right) \leq D$. Suppose now that there is a subsequence $\left\{\beta_{j_{k}}^{*}\right\}$ accumulating at one of the endpoints of $\beta^{*}$. By the Collar Lemma [3], the distance between $\beta^{*}$ and $\beta_{j}^{*}$ is bounded from below by a constant $d_{0}>0$. Then by Lemma 2 , we also obtain $l\left(\gamma_{j_{k}}^{*} ; \beta^{*}, \beta_{j_{k}}^{*}\right) \rightarrow \infty$ as $k \rightarrow \infty$. Again a contradiction to $l\left(\gamma_{j_{k}}^{*} ; \beta^{*}, \beta_{j_{k}}^{*}\right) \leq D$ for all $k$.

As we mentioned above, by the Collar Lemma, there exists $d_{0}>0$ such that the hyperbolic distance between $\beta^{*}$ and $\beta_{j}^{*}, j=1,2, \cdots, r$, is at least $d_{0}$. On the other hand, $l\left(\gamma^{*} ; \beta^{*}, \beta_{j}^{*}\right) \leq D$ for each $\gamma^{*}$ in $\mathcal{F}$. Let $d_{1}$ be the maximum of the 
hyperbolic distance between $\beta^{*}$ and $\beta_{j}^{*}$ for $j=1,2, \cdots, r$. Then $d_{0}<d_{1} \leq D$. For each $j=1,2, \cdots, r$, we can normalize the group $G_{0}$ so that $\beta^{*}=L_{-i, i}$ and $\beta_{j}^{*}=L_{\bar{b}, b}$, i.e., $\beta_{j}^{*}$ is the $d$-standard geodesic for some $d \in\left[d_{0}, d_{1}\right]$. Corresponding to each normalization, every map $F_{n}$ is changed to $F_{n, j}$. Let $s_{0}>0$ be the constant to guarantee the conclusion of Lemma 3. Choose $\delta>0$ so small that the conclusion of Lemma 4 follows. Recall that $F_{n}$ converges uniformly to the identity map. In fact, for each fixed $1 \leq j \leq r, F_{n, j}$ also converges uniformly to the identity map as $n \rightarrow \infty$. Thus we can choose $N(j)$ sufficiently large so that for each $n>N(j)$, $\left|F_{n, j}(x)-x\right|<\delta$. It follows from Lemma 4 that for every $n>N(j)$ and for every geodesic $\gamma^{*}=L_{p, q} \in \mathcal{F}$ crossing $L_{-i, i}$ and $L_{\bar{b}, b}$, we obtain

$$
\left|\log \frac{l\left(L_{p, q} ; L_{-i, i}, L_{\overline{b(d)}, b(d)}\right)}{l\left(L_{F_{n, j}(p), F_{n, j}(q)} ; L_{F_{n, j}(-i), F_{n, j}(i)}, L_{F_{n, j}(\bar{b}), F_{n, j}(b)}\right)}\right|<\epsilon .
$$

After applying the same argument to any geodesic $\beta_{j}^{*}$ for each $1 \leq j \leq r$ and then the same arguments of this part to any boundary component $B$ of $S_{0}$ (finitely many), we conclude that there exists $N_{3}$ such that for every $n>N_{3}$ and every $\gamma \in \Sigma_{S_{0}}^{\prime \prime}$ with $l_{S_{0}}(\gamma) \leq D$

$$
\left|\log \frac{l_{S_{n}}\left(f_{n}(\gamma)\right)}{l_{S_{0}}(\gamma)}\right|<\epsilon
$$

Letting $N=\max \left\{N_{1}, N_{2}, N_{3}\right\}$ and combining inequalities (3.3), (3.6) and (3.8), we obtain for every $n>N$,

$$
\log \sup _{\gamma \in \Sigma_{S_{0}}}\left\{\frac{l_{S_{n}}\left(f_{n}(\gamma)\right)}{l_{S_{0}}(\gamma)}, \frac{l_{S_{0}}(\gamma)}{l_{S_{n}}\left(f_{n}(\gamma)\right)}\right\}<\epsilon ;
$$

that is, $d_{M L}\left(\left[S_{n}, f n\right],\left[S_{0}\right.\right.$, id $\left.]\right) \rightarrow 0$ as $n \rightarrow \infty$. Thus the map

$$
\text { id : }\left(T\left(S_{0}\right), d_{T}\right) \rightarrow\left(T\left(S_{0}\right), d_{M L}\right)
$$

is continuous. result.

In fact, the techniques used in the proof of Theorem 5 also show the following

Corollary 2. Let $\left\{\left[S_{n}, f_{n}\right]\right\}$ be a sequence in $T\left(S_{0}\right)$ satisfying

(1) for each $n, f_{n}\left(\widetilde{S}_{0}\right)=\widetilde{S}_{n}$,

(2) $K\left(\left.f_{n}\right|_{\widetilde{S}_{0}}\right) \rightarrow 1$ as $n \rightarrow \infty$, and

(3) for each $n$, there is a lifting $F_{n}: \mathbf{D} \rightarrow \mathbf{D}$ of $f_{n}$ such that the sequence $\left\{F_{n}\right\}$ converges uniformly to the identity on $\mathbf{S}^{1}$.

Then $d_{M L}\left(\left[S_{n}, f_{n}\right],\left[S_{0}\right.\right.$, id $\left.]\right) \rightarrow 0$ as $n \rightarrow \infty$.

Unlike the case of $T^{R}\left(S_{0}\right)$, the metrics $d_{T}$ and $d_{M L}$ do not define the same topology on $T\left(S_{0}\right)$.

Theorem 6. There exists a sequence $\left\{\tau_{n}\right\}$ in $T\left(S_{0}\right)$ such that

$$
d_{M L}\left(\tau_{n}, \tau\right) \rightarrow 0 \quad \text { and } \quad d_{T}\left(\tau_{n}, \tau\right) \rightarrow \infty
$$

as $n \rightarrow \infty$, where $\tau=\left[S_{0}, \mathrm{id}\right]$.

Proof. For each $n=1,2,3, \cdots$, we construct a mapping $F_{n}: \mathbf{D} \rightarrow \mathbf{D}$ that projects to a mapping $f_{n}: S_{0} \rightarrow S_{0}$ such that the sequence $\left\{\left[S_{0}, f_{n}\right]\right\}$ satisfies the hypothesis of Corollary 2. 
Let $G_{0}$ be the Fuchsian group uniformizing $S_{0}$ and $\pi: \mathbf{D} \rightarrow S_{0}$ the covering map. Suppose $D_{0} \subseteq \mathbf{D}$ is a Dirichlet fundamental domain for $G_{0}$ and let $I$ be an arc contained in $\bar{D}_{0} \cap \mathbf{S}^{1}$ that projects to a segment on a boundary component of $S_{0}$. Let $T$ be a Möbius transformation from $\mathbf{D}$ onto the upper half plane that maps $I$ to the interval $[0,1]$, and for each $n$, let $b_{n}=1 /\left(2^{n+1}-1\right), c_{n}=1 / 2^{n}$. Define $\left.F_{n}\right|_{I}$ to be the mapping $T^{-1} \circ H_{n} \circ T$, where $H_{n}:[0,1] \rightarrow[0,1]$ is the piecewise linear map that sends $0, b_{n}, c_{n}$ and 1 to $0,\left(2^{n}-1\right) / 2^{2 n}$ and $c_{n}, 1$ respectively. Clearly, $H_{n}$ and hence $\left.F_{n}\right|_{I}$ converge to the identity map on $I$ uniformly as $n \rightarrow \infty$. Denote by $\beta$ the boundary geodesic on $S_{0}$ homotopic to the boundary component containing $\pi(I)$ and let $A$ be the connected component of $\bar{D}_{0} \backslash \pi^{-1}(\beta)$ containing $I$. Define $F_{n}$ to be the identity on $\left(\bar{D}_{0} \cap \mathbf{S}^{1} \backslash I\right) \cup\left(D_{0} \backslash A\right)$. Finally, foliate $A$ by geodesic rays starting at points in $D_{0} \cap \pi^{-1}(\beta)$ and ending at points in $\bar{A} \cap \mathbf{S}^{1}$. For every geodesic ray in the foliation starting at $z$ and ending at $x \in \mathbf{S}^{1}$, let $F_{n}$ map this ray onto the one starting at $z$ and ending at $F_{n}(x)$ by preserving the hyperbolic distance to $z$. The mapping $F_{n}: D_{0} \rightarrow D_{0}$ can be extended to the whole hyperbolic plane by pre-composing and post-composing by elements of $G_{0}$. Since $\left.F_{n}\right|_{\bar{D}_{0}}$ converges uniformly to the identity, it follows that $F_{n}: \mathbf{D} \rightarrow \mathbf{D}$ and $F_{n}: \mathbf{S}^{1} \rightarrow \mathbf{S}^{1}$ converge uniformly to the identity as well. Moreover, each $F_{n}$ can be projected to a map $f_{n}: S_{0} \rightarrow S_{0}$ such that $\left.f_{n}\right|_{\widetilde{S}_{0}}=\mathrm{id}_{\widetilde{S}_{0}}$.

Let $\tau_{n}=\left[S_{0}, f_{n}\right]$. Then by Corollary 2 ,

$$
d_{M L}\left(\tau_{n}, \tau\right) \rightarrow 0 \text { as } n \rightarrow \infty
$$

where $\tau=\left[S_{0}\right.$, id $]$.

For any four points $x, y, z, w \in \mathbf{R}$, let $\operatorname{cr}(x, y, z, w)$ denote the cross ratio

$$
\operatorname{cr}(x, y, z, w)=\frac{(y-x)(w-z)}{(z-y)(w-z)} .
$$

Notice that by construction,

$$
\operatorname{cr}\left(0, b_{n}, c_{n}, 1\right)=1
$$

and

$$
\operatorname{cr}\left(F_{n}(0), F_{n}\left(b_{n}\right), F_{n}\left(c_{n}\right), F_{n}(1)\right)=2^{n}-2+\frac{1}{2^{n-1}} \rightarrow \infty
$$

as $n \rightarrow \infty$. This implies that $K\left(f_{n}\right) \rightarrow \infty$ as $n \rightarrow \infty$, i.e.,

$$
d_{T}\left(\tau, \tau_{n}\right) \rightarrow \infty \text { as } n \rightarrow \infty .
$$

Theorems 5 and 6 together imply Theorem 2. Now we prove Theorem 3.

Proof. Let $G_{0}, D_{0}$ and $I=\lceil a, b\rceil$ be as in the proof of Theorem 6 . Let $T$ be a Möbius transformation from $\mathbf{D}$ onto the upper half plane $\mathbf{H}$ such that $I$ is mapped to $[0,1]$, with $T(a)=0$ and $T(b)=1$. For each $n$, we construct a mapping $F_{n}: \mathbf{H} \rightarrow \mathbf{H}$ in the same fashion as in the proof of Theorem 6 except that the map $H_{n}$ used to define $\left.F_{n}\right|_{I}=T^{-1} \circ H_{n} \circ T$ is replaced by the piecewise linear map that maps $0,1 / 2$ and 1 to $0,1 / 2^{n}$ and 1 , respectively. Note that for any $n>m$,

$$
H_{n} \circ H_{m}^{-1}(x)= \begin{cases}2^{m-n} x & \text { if } 0 \leq x \leq \frac{1}{2^{m}}, \\ \frac{2^{m}-2^{m+n}}{2^{n}-2^{n+m}}(x-1)+1 & \text { if } \frac{1}{2^{m}} \leq x \leq 1\end{cases}
$$

Thus

$$
\max _{x \in[0,1]}\left|H_{n} \circ H_{m}^{-1}(x)-x\right| \leq \frac{1}{2^{m}}-\frac{1}{2^{n}} \leq \frac{1}{2^{m}} .
$$


It follows that $H_{n} \circ H_{m}^{-1}$ is uniformly close to the identity if $n>m$ and $m$ is large, and hence so does $F_{n} \circ F_{m}^{-1}$. Let $f_{n}$ be the projection of $F_{n}$ to the surface $S_{0}$. Clearly, $f_{n} \circ f_{m}^{-1}$ is the identity on the Nielsen kernel $\widetilde{S}_{0}$. Now we apply Corollary 2 to conclude that

$$
d_{M L}\left(\left[S_{0}, f_{n}\right],\left[S_{0}, f_{m}\right]\right)=d_{M L}\left(\left[S_{0}, f_{n} \circ f_{m}^{-1}\right],\left[S_{0}, \mathrm{id}\right]\right) \rightarrow 0
$$

as $n, m \rightarrow \infty$. Thus $\left\{\left[S_{0}, f_{n}\right]\right\}$ is a Cauchy sequence under the metric $d_{M L}$. Now we show that this sequence cannot have a limit in $T\left(S_{0}\right)$ in this metric. Suppose not, then there is $\tau=[S, f] \in T\left(S_{0}\right)$ such that

$$
d_{M L}\left(\left[S_{0}, f_{n}\right],[S, f]\right) \rightarrow 0
$$

as $n \rightarrow \infty$. Then

$$
d_{L}\left(\left[S_{0}, f_{n}\right],[S, f]\right) \rightarrow 0
$$

in the reduced Teichmüller space $T^{R}\left(S_{0}\right)$ as $n \rightarrow \infty$.

Our construction shows that $\left[S_{0}, f_{n}\right]=\left[S_{0}\right.$, id $]$ in $T^{R}\left(S_{0}\right)$ for each $n$. It follows that $[S, f]$ and $\left[S_{0}\right.$, id] determine the same point in the reduced Teichmüller space and hence $S_{0}$ is conformally equivalent to $S$. By post-composing by an appropriate conformal mapping, we may assume that $\tau=\left[S_{0}, f\right]$ and $f_{n}$ is homotopic to $f$ for each $n$.

The maps $F_{n}$ are liftings of the maps $f_{n}$ and they agree with each other on the limit set of $G_{0}$. Let $F$ be the lifting of $f$ that agrees with $F_{n}$ on the limit set. Let $\beta_{1}^{*}$ and $\beta_{2}^{*}$ be two liftings of the boundary geodesic $\beta$ that is homotopic to the boundary component of $S_{0}$ to which $I=\lceil a, b\rceil$ projects. We can choose $I$ to be an interval such that it shares one endpoint with the common perpendicular geodesic $\gamma_{0}^{*}$ of $\beta_{1}^{*}$ and $\beta_{2}^{*}$. That is, we may assume $\gamma_{0}^{*}=L_{a, y}$ for some $y \in \mathbf{S}^{1}$.

By the construction, each $F_{n}$ fixes $a$ and $y$. Then $L_{a, y}=L_{F_{n}(a), F_{n}(y)}$ for all $n$. It follows that $l_{S_{0}}\left(f_{n}\left(\gamma_{0}\right)\right)=l_{S_{0}}\left(\gamma_{0}\right)$ for all $n$, where $\gamma_{0}$ is the projection of $\gamma_{0}^{*}$. Then, condition (3.9) implies $l_{S_{0}}\left(f\left(\gamma_{0}\right)\right)=l_{S_{0}}\left(\gamma_{0}\right)$. Since the common perpendicular is the unique shortest segment between $\beta_{1}^{*}$ and $\beta_{2}^{*}$, we must have $F(a)=a$ and $F(y)=y$.

Now let $\gamma_{1}^{*}=L_{T^{-1}(1 / 2), y}$. By the construction,

$$
F_{n}\left(T^{-1}(1 / 2)\right) \rightarrow a \text { as } n \rightarrow \infty .
$$

Thus

$$
l\left(L_{F_{n}\left(T^{-1}(1 / 2)\right), F_{n}(y)} ; \beta_{1}^{*}, \beta_{2}^{*}\right) \rightarrow l\left(L_{a, y} ; \beta_{1}^{*}, \beta_{2}^{*}\right) \text { as } n \rightarrow \infty
$$

that is

$$
l_{S_{0}}\left(f_{n}\left(\gamma_{1}\right)\right) \rightarrow l_{S_{0}}\left(\gamma_{0}\right) \text { as } n \rightarrow \infty,
$$

where $\gamma_{1}$ is the projection of $\gamma_{1}^{*}$. By condition (3.9), we obtain $l_{S_{0}}\left(f\left(\gamma_{1}\right)\right)=l_{S_{0}}\left(\gamma_{0}\right)$. Using again the uniqueness of the common perpendicular as the shortest segment between $\beta_{1}^{*}$ and $\beta_{2}^{*}$, we conclude that

$$
F\left(T^{-1}(1 / 2)\right)=a .
$$

Since $F(a)=a$, we obtain a contradiction to the injectivity of $F$ on $\overline{\mathbf{D}}$. 


\section{References}

[1] Abikoff, W.: The real analytic theory of Teichmüller space. - Lecture Notes in Math. 820, Springer-Verlag, Berlin-Heidelberg-New York, 1980.

[2] Bers, L.: Nielsen extension of Riemann surfaces. - Ann. Acad. Sci. Fenn. Ser. A I Math. 2, 1976, 29-34.

[3] Keen, L.: Collars on Riemann surfaces. - In: Discontinuous groups and Riemann Surfaces, Proceedings of the Maryland Conf. 1973, Ann. of Math. Stud. 79, 1974, 263-268.

[4] Leнto, O.: Univalent functions and Teichmüller spaces. - Grad. Texts in Math. 109, SpringerVerlag, New York, 1987.

[5] LI, Z.: Teichmüller metric and length spectrum of Riemann surfaces. - Scientia Sinica Ser. A $24,1986,265-274$.

[6] LI, Z.: Length spectrum of Riemann surfaces and Teichmüller metric. - Bull. London Math. Soc. $35,2003,247-254$.

[7] LiU, L.: On the length spectrums of non-compact Riemann surfaces. - Ann. Acad. Sci. Fenn. Math. 24, 1999, 11-22.

[8] Shiga, H.: On a distance defined by the length spectrum on Teichmüller space. - Ann. Acad. Sci. Fenn. Math. 28, 2003, 315-326.

[9] Sorvali, T.: The boundary mapping induced by an isomorphism of covering groups. - Ann. Acad. Sci. Fenn. Ser. A I Math. 526, 1972, 1-31.

[10] Sorvali, T.: On Teichmüller spaces of tori. - Ann. Acad. Sci. Fenn. Ser. A I Math. 1, 1975, $7-11$.

Received 12 March 2013 • Accepted 10 January 2014 